\title{
Exploring the Occurrence
} Characteristics of Microplastics in Typical Maize Farmland Soils With Long-Term Plastic Film Mulching in Northern China

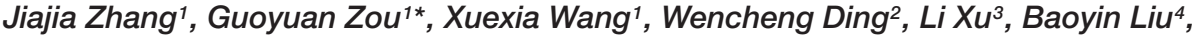
Yunsen $\mathbf{M u}^{5}$, Xuran Zhu ${ }^{5}$, Lianjie Song ${ }^{5}$ and Yanhua Chen ${ }^{1 *}$

1 Institute of Plant Nutrition, Resources and Environment, Beijing Academy of Agriculture and Forestry Sciences, Beijing, China, ${ }^{2}$ Ministry of Agriculture and Rural Affairs Key Laboratory of Plant Nutrition and Fertilizer, Institute of Agricultural Resources and Regional Planning, Chinese Academy of Agricultural Sciences, Beijing, China, ${ }^{3}$ Beijing Research Center for Agricultural Standards and Testing, Beijing Academy of Agriculture and Forestry Sciences, Beijing, China, ${ }^{4}$ Pingquan Municipal Bureau of Agriculture and Rural Affairs, Chengde, China, ${ }^{5}$ Chengde Academy of Agriculture and Forestry Sciences, Chengde, China
\end{abstract}

OPEN ACCESS

Edited by:

Xuetao Guo,

Northwest A\&F University, China

Reviewed by:

Xiaomei Yang,

Wageningen University \& Research,

Netherlands

Ninging Song,

Qingdao Agricultural University, China Jianying Shang,

China Agricultural University, China

*Correspondence:

Guoyuan Zou

gyzou@163.com

Yanhua Chen

yhchen55@126.com

Specialty section:

This article was submitted to

Marine Pollution,

a section of the journal

Frontiers in Marine Science

Received: 22 October 2021 Accepted: 17 November 2021 Published: 09 December 2021

Citation:

Zhang J, Zou G, Wang X, Ding W, $X u L$, Liu B, Mu Y, Zhu X, Song L and Chen $Y$ (2021) Exploring the Occurrence Characteristics of Microplastics in Typical Maize Farmland Soils With Long-Term Plastic Film Mulching in Northern China. Front. Mar. Sci. 8:800087. doi: 10.3389/fmars.2021.800087
Microplastics pollution has been threatening the global environmental security, in which agricultural activities are considered as a main source of microplastics occurrence in soils. However, little is known about the occurrence characteristics of microplastics in agricultural soils with long-term plastic film mulching. Therefore, the abundance, distribution, and composition of microplastics were investigated by analyzing 225 soil samples collected from typical maize (Zea mays L.) planting zones with and without long-term (>20 years) plastic film mulching in northern China. Microplastics abundance in mulched soils (754 \pm 477 items $\mathrm{kg}^{-1}$ ) was significantly higher than that in nonmulched soils (376 \pm 149 items $\mathrm{kg}^{-1}$ ), which indicated that plastic film mulching contributed half of microplastics in soils. Moreover, microplastics abundance was significantly positively related to the length of time with film mulching applied. The percentage of microplastics $<0.5 \mathrm{~mm}$ in mulched soils $(50.9 \%)$ was significantly lower than that in non-mulched soils (62.2\%). Microplastics abundance and size in mulched and non-mulched soils decreased with increased soil depth. Most microplastics were fragments of polypropylene, films of polyethylene, and fibers of polyester. The proportion of films in mulched soils was significantly higher than in non-mulched soils, whereas that of fibers was significantly higher in non-mulched soils. This study confirmed that long-term plastic film mulching increases microplastics pollution in agricultural soils, warranting further evaluation of the associated ecological risks of microplastics in soil ecosystems.

Keywords: microplastics, maize, plastic film mulching, farmland soils, distribution characteristics

\section{INTRODUCTION}

Global plastic production has increased from 230 to 359 million tons between 2005 and 2018, and China accounted for $30 \%$ of that production (Statista, 2020). However, with recycling rates that are generally low, plastic waste is considered a global environmental pollution issue because of its low degradability (Barboza et al., 2018). Although plastics can remain in the environment permanently, 
they decompose into increasingly smaller pieces of plastic debris under the actions of biological, physical, and chemical processes, such as decomposition by intestinal microorganisms and insects, agriculture cultivation, weathering, and oxidative degradation under ultraviolet irradiation (Sul and Costa, 2014; Rillig et al., 2017; Ahmed et al., 2018). Microplastics are plastic particles $<5 \mathrm{~mm}$, and because of their small size and large quantities, they are widely distributed in freshwater, marine, terrestrial, and other environments (Peng et al., 2018; Sighicelli et al., 2018; Gong and Xie, 2020). As new environmental pollutants, microplastics have received special attention and are listed as the second most important scientific issue in the ecology and environmental science (Farrell and Nelson, 2013; Horton et al., 2017). Much research has been conducted on the occurrence, fate, and effects of microplastics in aquatic environments (Vaughan et al., 2017; Tang et al., 2018; Wang and Wang, 2018). Although soils are the major and direct sources of microplastics, much remains unknown regarding microplastics in terrestrial environments (Rillig, 2012; Horton et al., 2017).

The major sources of microplastics in soils include residues of agricultural plastic film, sludge and sewage application, wastewater irrigation, organic fertilizer application, surface runoff, and atmospheric deposition (Zubris and Richards, 2005; Bläsing and Amelung, 2018; He et al., 2018; Zhang J. J. et al., 2021). The annual discharge of microplastics to terrestrial systems is 4 to 23 times that to the marine systems (Horton et al., 2017). When discharged from agricultural or industrial production activities, microplastics can remain in soils and be directly absorbed by various soil organisms, posing a threat to their reproduction and growth and accumulating in food chains and causing damage to soil biota in different trophic levels (Rillig, 2012; Rillig and Lehmann, 2020). In agricultural soils of Northwest China, the concentrations of microplastics range from 1,430 to 3,410 items $\mathrm{kg}^{-1}$ (Ding et al., 2020). According to Yu et al. (2020), the abundance of microplastics in vegetable farmland soils of northern China averaged 1,444 items $\mathrm{kg}^{-1}$, ranging from 310 to 5,698 items $\mathrm{kg}^{-1}$. Plant roots [i.e., wheat (Triticum aestivum L.) and lettuce (Lactuca sativa L.)] can absorb micron and submicronsized microplastics through specific crack-entry modes and then transport the microplastics to shoots (Li et al., 2020). In addition, some microplastics migrate horizontally to rivers (in runoff), the atmosphere, or other parts of the land through the actions of wind and water, whereas others remain in the soil and migrate vertically to deeper soil (Huerta Lwanga et al., 2016; Scheurer and Bigalke, 2018). Microplastics can also absorb pollutants in soils, including antibiotics, pesticides, heavy metals, and persistent organic pollutants. These pollutants can cause serious toxic effects when transported into organisms, increasing the long-term harmful effects (Avio et al., 2015; Hüffer et al., 2018). Therefore, microplastics may threaten soil ecological functions and adversely affect food production (Turner and Holmes, 2015; Li et al., 2018). Because of the negative effects of microplastics on soil ecosystems, it is urgent to identify the distribution and characteristics of microplastics pollution in soils in order to maintain ecological security, promote green development, and ultimately implement effective mitigation and prevention measures.

However, most research on microplastics has focused on ocean and water pollution (Sul and Costa, 2014; Vaughan et al., 2017; Peng et al., 2018). Although microplastics have been investigated in soils of flood zones, industrial areas, vegetable fields, facility agricultural soils and in riverine soils, little research has been conducted in soils with grain crops (Fuller and Gautam, 2016; Scheurer and Bigalke, 2018; Amrutha and Warrier, 2020; Chen et al., 2020). Maize (Zea mays L.) is the largest grain crop in China, and Hebei Province is one of the main provinces for maize production (Zhang et al., 2017). Low accumulated temperature and little rain are the main factors limiting high maize yields in the northern of the province. Therefore, to overcome these constraints, the cultivation techniques of agricultural plastic film mulching have been adopted for more than 20 years. Long-term applications of plastic mulching can result in large amounts of plastic debris remaining in farmland soils that gradually decompose into smaller-sized fragments and form microplastics (Astner et al., 2019). However, the pollution status of microplastics in maize farmland soils that use plastic film mulching is unknown. The authors hypothesized that accumulated microplastics in soils with long-term plastic film mulching would be higher than that in non-mulched farmland soils because of the fragmentation of residual films, and microplastics size and shape would also be significantly different. Therefore, this systematical study was conducted with the purpose of investigating the distribution and characteristics of microplastics pollution in mulched and non-mulched maize farmland soils in northern China. The abundance, size, and others that indicate possible sources of microplastics such as polymer composition, color, and shape were determined.

\section{MATERIALS AND METHODS}

\section{Study Area and Soil Sample Collection}

Pingquan City $\left(40^{\circ} 24^{\prime} 00^{\prime \prime} \mathrm{N}\right.$ to $40^{\circ} 40^{\prime} 17^{\prime \prime} \mathrm{N}, 118^{\circ} 21^{\prime} 03^{\prime \prime} \mathrm{E}$ to $\left.119^{\circ} 15^{\prime} 34^{\prime \prime} \mathrm{E}\right)$ is in the northeast part of Hebei Province and at the subsidence zone at the eastern end of the Yanshan Mountains. The elevation of the planting area is 450 to $800 \mathrm{~m}$. The city has a continental monsoon climate. Springs are dry with little rain, summers are rainy and hot, autumns are cool with drought, and winters are cold with little snow. The average annual precipitation is $523 \mathrm{~mm}$, and the average air temperature is $7.3^{\circ} \mathrm{C}$. The frost-free period is 110 to $125 \mathrm{~d}$ per year, and the accumulated temperature is 2500 to $2850^{\circ} \mathrm{C}\left(\geq 10^{\circ} \mathrm{C}\right)$. Maize is the main food crop in suburban farmlands and the most important source of agricultural products for circumjacent inhabitants. Maize is monocropped in Pingquan City. In the northern part of the city, due to low accumulated temperature and little rain, the cultivation techniques of agricultural plastic film mulching have been adopted for more than 20 years. However, plastic film mulching has never been used in the southern part. Maize is sown in late April to early May and harvested in late September to early October. In Pingquan City, the total area planted to grain crops in 2019 was 
$40.81 \times 10^{3} \mathrm{ha}$, and the annual production was $210.67 \times 10^{3}$ t. Maize accounted for $96.0 \%$ of the total area planted and $91.5 \%$ of the annual yield. As of 2019, the annual quantity of agricultural plastic film for maize planting in the city exceeded 50 tons and covered approximately $7 \times 10^{3}$ ha (Hebei Statistics Bureau, 2020). The thickness of plastics films used in this area generally ranged from 0.004 to $0.006 \mathrm{~mm}$. Local farmers usually collect the plastic film after maize harvest, with a recycling rate of 70 to $80 \%$.

Soil samples were collected in October 2020 from 15 villages, each with $10 \mathrm{~km}^{2}$ of farmland planted to maize. Ten villages had mulched farmland soils and five had nonmulched farmland soils (Supplementary Figure $\mathbf{1}$ and Table 1). Five representative sampling sites were selected from the maize farmlands of each village, and the soil samples from each site were composed of three subsamples. In these sites, farmland soils had broken plastic films on the surface and were surrounded by discarded plastics (e.g., polyfoams, nylon nets, plastic bags, and other domestic garbage) (Supplementary Figure 2). In each sampling site, soil samples were collected from the arable layer in a $0.5 \times 0.5 \mathrm{~m}$ area using a stainlesssteel shovel. Sampling was stratified, and soils were collected at $0-10,10-20$, and $20-30 \mathrm{~cm}$ depths, with depths measured accurately with calipers. Therefore, fifty and twenty-five sites were sampled in mulched and non-mulched farmland soils, respectively, and with the three depths, a total of 150 and 75 samples were obtained from the mulched and non-mulched farmland soils, respectively. After removing large visible garbage $(>5 \mathrm{~cm})$, approximately $2 \mathrm{~kg}$ of soil was collected from each soil layer in each sampling site. All soil samples were stored in labeled aluminum boxes and then transferred to the laboratory for analyses.

\section{Sampling Processing}

Microplastics were extracted from soil samples by a modified density separation method with saturated $\mathrm{NaCl}$ solution $(1.2 \mathrm{~g}$ $\mathrm{cm}^{-3}$ ) that has been demonstrated successfully applied for agricultural soils (Scheurer and Bigalke, 2018; Lv et al., 2019; Wang et al., 2020; Li et al., 2021). Specifically, the soil samples were air-dried and sieved through a $5-\mathrm{mm}$ stainless steel sieve to remove plant residues and stones (or large debris). Airdried soil subsamples of $50 \mathrm{~g}$ were weighted and mixed with $200 \mathrm{~mL}$ of saturated $\mathrm{NaCl}$ solution in $250 \mathrm{~mL}$ clean and dry glass bottles. The mixtures were stirred thoroughly for $30 \mathrm{~min}$ and then kept standing for $24 \mathrm{~h}$. After that, the supernatants were collected in pre-cleaned beakers. To fully extract microplastics from soils, the density separation process was repeated three times. Finally, all collected supernatants were filtered through $0.45 \mu \mathrm{m}$ GF/A membranes (Whatman, United States). All substances on filter papers were washed with $50 \mathrm{~mL}$ of $30 \% \mathrm{H}_{2} \mathrm{O}_{2}$ solution into $100 \mathrm{~mL}$ glass beakers to digest organic matters, and the beakers were placed on a graphite electric heating plate for digestion at $60^{\circ} \mathrm{C}$ for $24 \mathrm{~h}$. After digestion, the solutions were filtered through $0.45 \mu \mathrm{m} \mathrm{GF} / \mathrm{A}$ membranes and rinsed with deionized water. Finally, all filter papers with attached microplastics were put into glass petri dishes and dried at room temperature. Filter papers were observed under a microscope.

\section{Observation and Identification of Microplastics}

Microplastics on filter papers were inspected visually using a stereomicroscope (SZ760-DM500). In order to reduce the possibility of microplastics misidentification, the classification criteria developed by Hidalgo-Ruz et al. (2012) and Peng et al. (2017) were used in the visual identification. The particles with clear shapes and colors were selected and identified as potential microplastics according to the criteria. While other particles that were difficult to be identified but had similar shapes were considered to be suspected microplastics. All these particles were hand-sorted using stainless steel finetip tweezers under the stereomicroscope, and the suspected particles were stored separately. The number of these particles were counted (items $\mathrm{kg}^{-1}$ ), and the shapes, sizes, and colors were recorded. To separate microplastics from other materials, the polymer composition was determined by a microFourier Transform Infrared Spectrometer ( $\mu$-FTIR, Spotlight 400, PerkinElmer, United States). The spectrum ranging from 650 to $4,000 \mathrm{~cm}^{-1}$ was used to scan samples 32 times at a resolution of $4 \mathrm{~cm}^{-1}$ in transmission mode. The spectra of all selected samples were identified using the Spectrum software and compared with the standard spectral libraries of polymers (i.e., Sadtler Infrared Spectral library) to determine the polymer compositions. When the matching degree exceeded $70 \%$, the sample corresponding to the spectrum was considered microplastics, and the corresponding polymer was confirmed accordingly (Liu et al., 2018).

\section{Quality Assurance and Quality Control in Experiments}

To avoid potential plastic pollution of artificial and airborne and ensure the reliability of the experimental results, strict quality control was performed during the experiment. All plastic materials were avoided during the entire pretreatment process, and all materials used for analysis were made of glass (e.g., stirrer rods, funnels, and petri dishes). All equipment and containers were carefully rinsed with deionized water for more than three times, and all samples were always sealed with aluminum foil between separate experimental steps to minimize possible pollution from indoor air. During the entire experiment, researchers wore cotton lab coats and gloves. In addition, blank experiments were performed to check the possible pollution of ultrapure water and air in the laboratory during the operation. No microplastics were detected in the blank controls.

A recovery experiment was performed to verify the separation method with saturated $\mathrm{NaCl}$ solution. Some commercial polyethylene (PE), polypropylene (PP), and polystyrene (PS) plastic products were selected and ground into microplastic particles (200 to $2000 \mu \mathrm{m}$ ) with a mill in the laboratory. A 50g relative clean soil was mixed with 30 counts of the polymer 
TABLE 1 | Abundance statistics of microplastics at different soil layers in mulched and non-mulched farmlands in Pingquan City, Hebei Province, China.

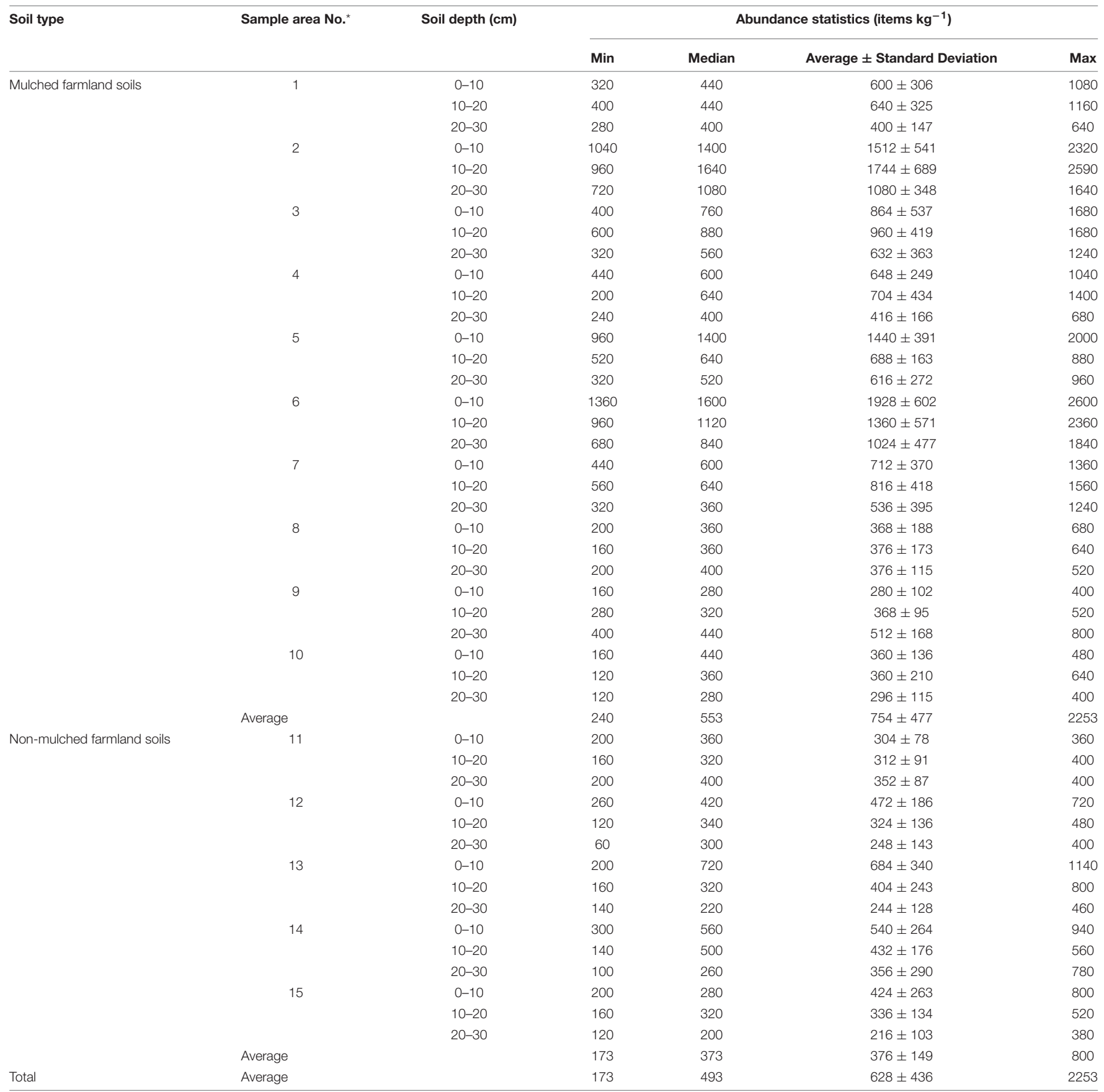

${ }^{*}$ Each area included five sampling sites.

particles. The mixture was pretreated and analyzed using the separation method as described above. Five replicates were settled for each type of microplastic. The recovered MP particles were counted under a stereomicroscope and the recovery rates for the MP particles were above $90 \%$.

\section{Statistical Analyses}

Statistical analyses were performed using SPSS software (Version 23.0, IBM). The figures were plotted using Microsoft Excel (2016), SigmaPlot (14.0), and R ("ggplot2" package, version
3.3.3). Independent samples $t$-test was adopted to determine the differences in microplastics abundances, sizes, shapes, and colors between the mulched and non-mulched farmland soils at the $95 \%$ confidence level. The PROC GLM procedure was used to determine the differences in particle size of microplastics among different soil layers in the mulched and non-mulched farmland soils. One-way ANOVA was used to determine the differences in microplastics abundances among different soil layers and the differences in the percentages of microplastics among different soil layers in each size range in the mulched 
and non-mulched farmland soils. Average values were compared using the least significant difference (LSD) test at $p<0.05$. Linear regression analysis was used to quantify the relationship between the microplastics abundances and the length of time with film mulching applied.

\section{RESULTS}

\section{Abundance and Vertical Distribution of Microplastics in Maize Farmland Soils}

Microplastics were detected in different soil layers at all sampling sites. On average, the microplastics abundance of $754 \pm 477$ items $\mathrm{kg}^{-1}$ (range: 240 to 2,253 items $\mathrm{kg}^{-1}$ ) in mulched farmland soils was significantly higher than the abundance of $376 \pm 149$ items $\mathrm{kg}^{-1}$ (range: 173 to 800 items $\mathrm{kg}^{-1}$ ) in non-mulched farmland soils ( $p<0.05$, Figure $\mathbf{1 A}$ and Table $\mathbf{1})$.

Average abundances of microplastics at $0-10 \mathrm{~cm}, 10-20 \mathrm{~cm}$, and $20-30 \mathrm{~cm}$ in mulched farmland soils were $871 \pm 641$ items $\mathrm{kg}^{-1}$ (range: 160 to 2,600 items $\mathrm{kg}^{-1}$ ), $802 \pm 561$ items $\mathrm{kg}^{-1}$ (range: 120 to 2,600 items $\mathrm{kg}^{-1}$ ), and $589 \pm 363$ items $\mathrm{kg}^{-1}$ (range: 120 to 1,840 items $\mathrm{kg}^{-1}$ ), respectively (Figure 1B). In non-mulched farmland soils, at the same depths, the abundances were $485 \pm 257$ items $\mathrm{kg}^{-1}$ (range: 200 to 1,140 items $\mathrm{kg}^{-1}$ ), $362 \pm 158$ items $\mathrm{kg}^{-1}$ (range: 120 to 800 items $\mathrm{kg}^{-1}$ ), and $283 \pm 164$ items kg-1 (range: 60 to 780 items kg-1), respectively. The vertical distributions of microplastics in mulched and nonmulched farmland soils were similar. Microplastics abundance in the two farmland soils decreased gradually with the increase in soil depth, and the abundance at $0-10 \mathrm{~cm}$ was significantly greater than that at $20-30 \mathrm{~cm}$ ( $p<0.05$, Figure 1B). Of all sampling areas, the highest concentration of microplastics was 2,600 items $\mathrm{kg}^{-1}$ at $0-10 \mathrm{~cm}$ in area 6 of the mulched farmland soils (Table 1). The lowest abundance of microplastics was only 60 items $\mathrm{kg}^{-1}$ at $20-30 \mathrm{~cm}$ in area 12 of the nonmulched farmland soils. In addition, microplastics abundance was significantly positively related to the length of time with film mulching applied $\left(\mathrm{R}^{2}=0.43, p<0.05\right)$ (Figure 2).

\section{Size Distribution of Microplastics}

The size of microplastics particles ranged from 0.03 to $5 \mathrm{~mm}$. The size distribution of microplastics in different soil layers in mulched and non-mulched farmland soils was generally similar, and the percentage of size distribution decreased with the increase of particle size (Figures 3A,C and Supplementary Table 2). In mulched soils, $50.9 \%$ of the particles were $<0.5 \mathrm{~mm}$, $18.5 \%$ were $0.5-1.0 \mathrm{~mm}, 17.9 \%$ were $1.0-2.0 \mathrm{~mm}$, and $12.6 \%$ were $2.0-5.0 \mathrm{~mm}$. In non-mulched soils, $62.2 \%$ of the particles were $<0.5 \mathrm{~mm}, 13.3 \%$ were $0.5-1.0 \mathrm{~mm}, 12.8 \%$ were $1.0-$ $2.0 \mathrm{~mm}$, and $11.6 \%$ were $2.0-5.0 \mathrm{~mm}$. The percentage of microplastics $<0.5 \mathrm{~mm}$ in mulched soils was significantly lower than that in non-mulched soils (Figure 3E). In the mulched and non-mulched farmland soils, the highest percentage of microplastics $<0.5 \mathrm{~mm}$ was at $20-30 \mathrm{~cm}$, with averages of $57.9 \pm 9.0 \%$ and $66.1 \pm 9.8 \%$, respectively. The percentage of microplastics $<0.5 \mathrm{~mm}$ was significantly different between $0-10$ and $20-30 \mathrm{~cm}$ in mulched farmland soils ( $p<0.05$, Figure $3 \mathbf{A})$.
For the particles $2.0-5.0 \mathrm{~mm}$, their percentages tended to decrease with the increase of soil depth in mulched and non-mulched farmland soils, and the percentage at $20-30 \mathrm{~cm}$ was significantly lower than that at $0-10 \mathrm{~cm}$ in non-mulched farmland soils $(p<0.05$, Figure 3C).

In mulched soils, the average size of microplastics was $1.03 \pm 1.08 \mathrm{~mm}, 0.83 \pm 0.85 \mathrm{~mm}$, and $0.82 \pm 0.89 \mathrm{~mm}$ at $0-$ $10 \mathrm{~cm}, 10-20 \mathrm{~cm}$, and $20-30 \mathrm{~cm}$, respectively. In non-mulched soils, the average size was $1.07 \pm 1.27 \mathrm{~mm}, 0.86 \pm 0.87 \mathrm{~mm}$, and $0.78 \pm 0.72 \mathrm{~mm}$ at $0-10 \mathrm{~cm}, 10-20 \mathrm{~cm}$, and $20-30 \mathrm{~cm}$, respectively (Figures 3B,D). The average size of microplastics at $0-10 \mathrm{~cm}$ in the two farmland soils was significantly larger than that at $10-20 \mathrm{~cm}$ and $20-30 \mathrm{~cm}(p<0.05$, Figures 3B,D).

\section{Shape Characteristics of Microplastics}

Four shapes of microplastics were detected: fragment, fiber, film, and pellet (Figure 4). The distributions of microplastics shapes in mulched and non-mulched farmland soils were different (Figures 5A,B). Fragments were the highest percentage (37.7\%) of microplastics in mulched and non-mulched soils, followed by films $(33.9 \%)$, fibers $(21.9 \%)$, and pellets $(6.6 \%)$ in mulched soils, and fibers $(29.6 \%)$, films $(24.3 \%)$, and pellets $(4.2 \%)$ in non-mulched soils. The percentage of films in mulched soils was significantly higher than that in non-mulched soils, whereas that of fibers was significantly higher in non-mulched soils $(p<0.05$, Figure 5C). Fragments, fibers, and films were found in all soil samples, whereas pellets were detected in $70.0 \%$ of mulched soils and $60.0 \%$ of non-mulched soils (Supplementary Table 2).

\section{Color Characteristics of Microplastics}

Five colors of microplastics were detected: white, transparent, yellow, blue, and black. Overall, the distributions of different colors of microplastics at different soil depths in mulched and non-mulched soils were similar (Figures 6A,B and Supplementary Table 2). Most microplastics were white in mulched $(40.2 \%)$ and non-mulched $(43.3 \%)$ soils, followed by those that were transparent (36.4 and 39.5\%, respectively), yellow (16.7 and $11.8 \%$, respectively), and blue (4.4 and 3.2\%, respectively). Black microplastics were detected at the lowest frequency, with average percentages of only 2.2 and $1.6 \%$ in mulched and non-mulched soils, respectively. No significant difference was observed in the proportion of different colors between mulched and non-mulched soils.

\section{Polymer Composition of Microplastics}

The polymer composition of microplastics included polyethylene $(\mathrm{PE})$, polypropylene (PP), polyester, poly(ethylene terephthalate) (PET), rayon, poly(ethyl acrylate) (PEA), cellophane, and polyacrylonitrile/acrylic acid (PAN) (Figure 7A). Of the microplastics, $36.9 \%$ were composed of PP, $26.5 \%$ of $\mathrm{PE}, 17.1 \%$ of polyester, $10.9 \%$ of PET, $4.7 \%$ of rayon, $1.8 \%$ of PEA, $1.2 \%$ of cellophane, and $0.9 \%$ of PAN. Notably, the dominant polymer types were PP, PE, and polyester.

The polymer composition of microplastics varied with shape (Figure 7B). Polypropylene was the dominant polymer type in fragments, accounting for $66.4 \%$ of the total polymers, followed by PE $(32.7 \%)$ and PEA (0.9\%). The polymer types in pellets 

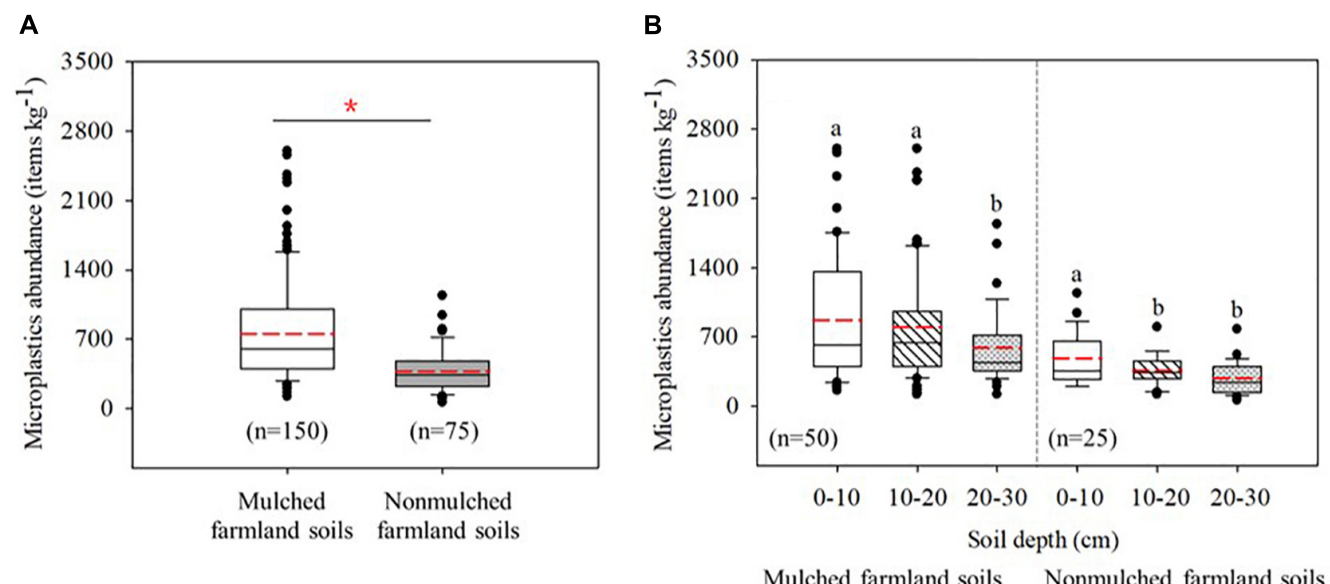

FIGURE 1 | Abundances of microplastics (items $\mathrm{kg}^{-1}$ ) in mulched and non-mulched farmland soils. (A) Total abundance ( $\left.{ }^{\star} p<0.05\right)$. (B) Abundances at different soil depths $(\mathrm{cm})$, with different letters indicating significant differences at $p<0.05$. Box-whisker diagrams show the average, 25 th, 50 th, 75 th percentiles.

included PE (53.5\%), PP (28.6\%), and PEA (17.9\%), whereas in films, only $\mathrm{PE}(67.4 \%)$ and $\mathrm{PP}(32.6 \%)$ were identified. In fibers, polyester $(36.7 \%)$ was the dominant polymer, follow by PET (23.4\%), PP (19.0\%), rayon (10.1\%), PE (6.3\%), cellophane (2.5\%), and PAN (1.9\%).

\section{DISCUSSION}

\section{Abundance and Vertical Distribution of Microplastics in Maize Farmland Soils}

This study confirmed the occurrence of microplastics in maize farmland soils with long-term plastic film mulching in northern China. The abundance of microplastics in mulched soils was significantly higher than that in non-mulched soils (Figure 1A). This apparent distinction can probably be ascribed to the application of plastic mulching. Moreover, microplastics abundance was also different among the mulched farmlands,

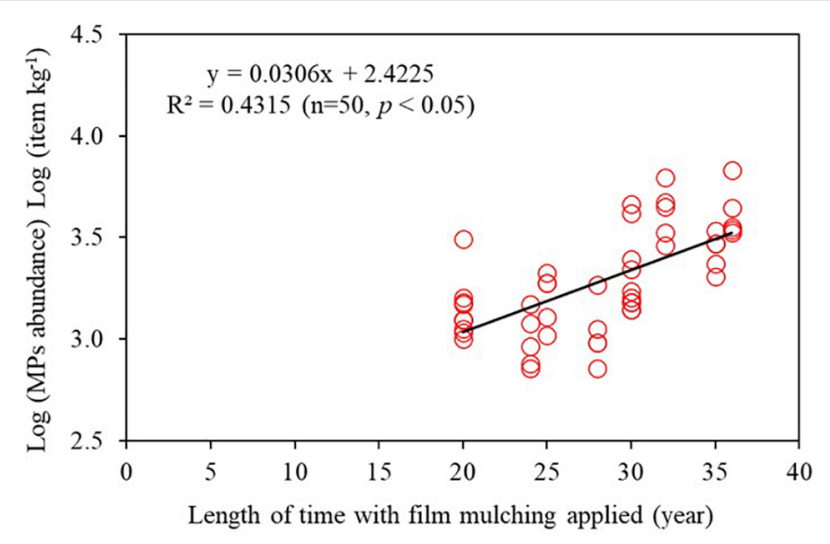

FIGURE 2 | Correlation between the length of time with film mulching applied and microplastics abundance. with the differences primarily due to the length of time with film mulching applied. A significant positive linear correlation was obtained between microplastics abundance and the length of time with film mulching applied (correlation coefficient $\mathrm{R}^{2}=0.58, p<0.05$, Figure 2). For example, area 6 had the highest concentration of microplastics (i.e., 2,600 items $\mathrm{kg}^{-1}$ ) because of the longest period of plastic mulching (over 35 years) (Table 1). This was consistent with Huang et al. (2020) who found that microplastics abundances substantially increased with plastic mulching continuously employed. These results indicated that microplastics abundance increased greatly with long-term application of plastic films. In addition, a certain amount of microplastics (mainly fragments and fibers) was detected in non-mulched soils, which has no mulching history, indicating that plastic film mulch is not the only source of microplastics in agricultural soils. In the sampling areas, the residues of plastic package bags, pesticide bottles, broken fertilizer packaging bags, and residual agricultural film were found whether film mulching was used or not that could be also a potential source of microplastics. Apart from these, atmospheric deposition is also a route of fibrous microplastics entering soils (Bläsing and Amelung, 2018). All of these can lead to the appearance of microplastics in non-mulched soils.

To better understand the pollution status of microplastics in maize farmland soils, the results of this study were compared with the characteristics of microplastics in different terrestrial systems that were summarized in the current study (Supplementary Table 3). The average abundance of microplastics (i.e., 628 items $\mathrm{kg}^{-1}$ ) in this study is comparable with that in Hebei coastal soils (i.e., 634 items $\mathrm{kg}^{-1}$ ) and Swiss floodplain soils (i.e., 593 particles $\mathrm{kg}^{-1}$ ) (Zhou et al., 2016; Scheurer and Bigalke, 2018). However, microplastics concentration in maize farmland soils is relatively high compared with that in other soil types, including greenhouse and orchard soils, rice-fish co-culture system soil, agricultural soils amended with pig manure, and riverine soils (Zhang et al., 2018; Lv et al., 2019; Amrutha and Warrier, 2020; Yang et al., 2020). However, the microplastics 


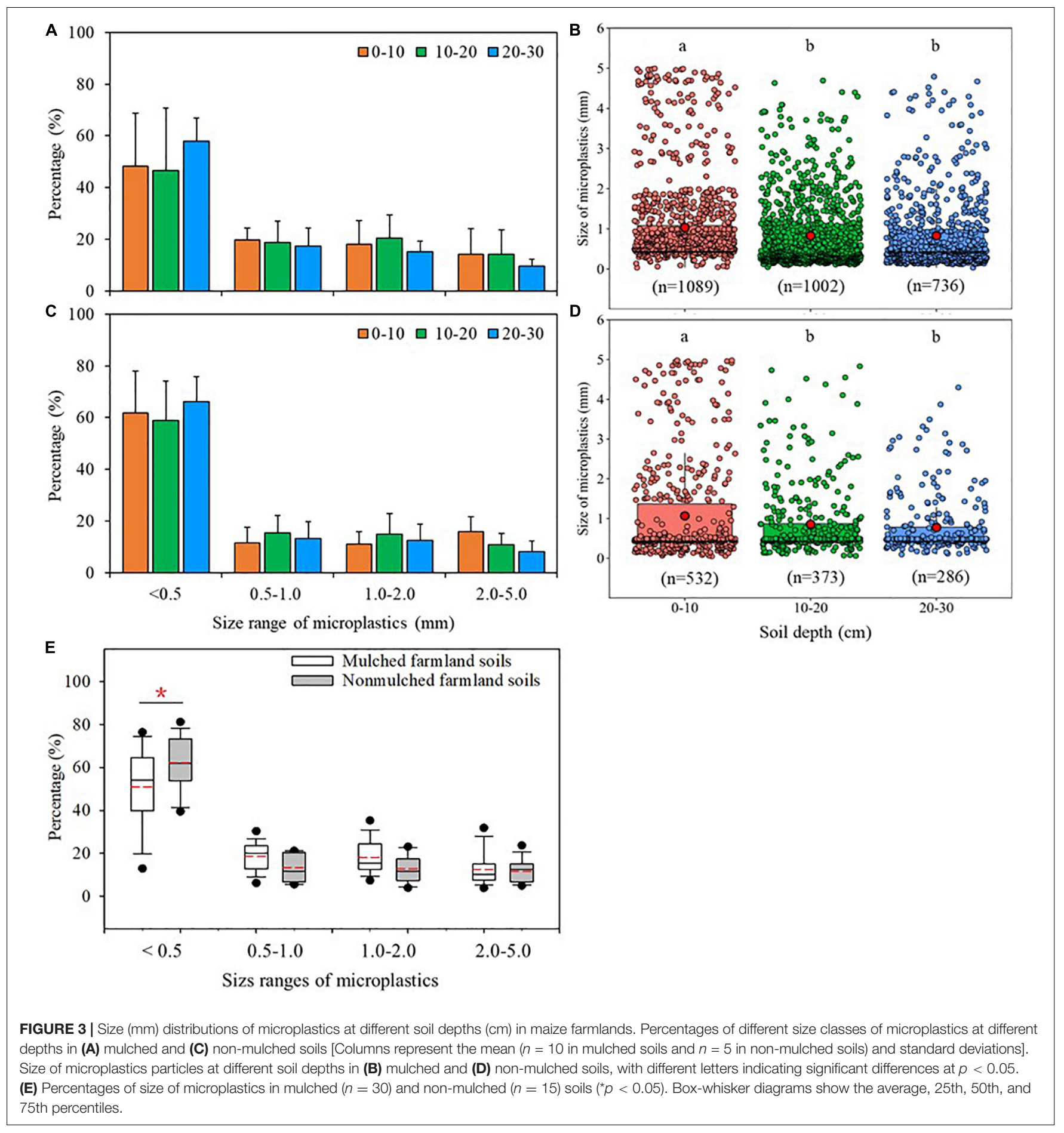

abundance in this study is much lower than that in facility agricultural soils and vegetable farmland soils (Zhang and Liu, 2018; Chen et al., 2020). Therefore, as demonstrated by the above studies, microplastics are common contaminants in soil systems. Although most soils were collected from farmlands, the concentrations of microplastics varied widely in different regions, which may be due to many factors, such as crop rotation, fertilization, tillage, sampling sites, and extraction methods of microplastics in soils (Chen et al., 2020). In addition, the distribution of microplastics pollution is also associated with pollution sources and plastics content (Chen et al., 2020). Moreover, this study found that microplastics abundances in both mulched and non-mulched soils decreased gradually with increased soil depth (Figure 1B). During the maize planting in sampling areas, soils are usually ploughed once every two years in mulched farmlands and once per year in non-mulched farmlands, which might contribute to the vertical transfer of microplastics between shallow and deep soil 
A

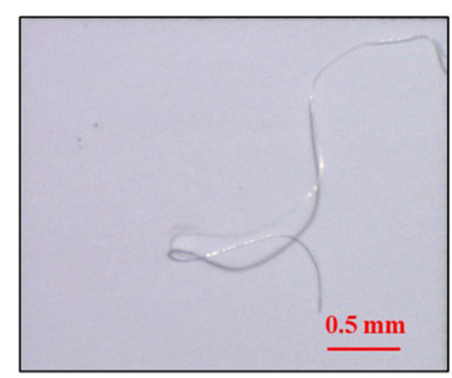

D

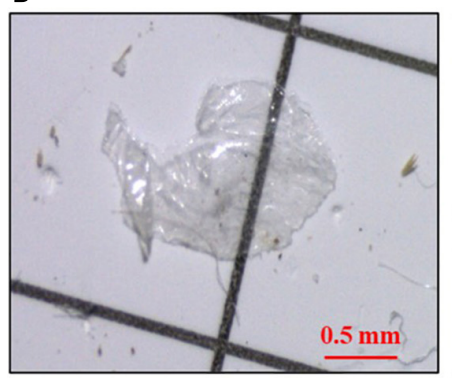

B

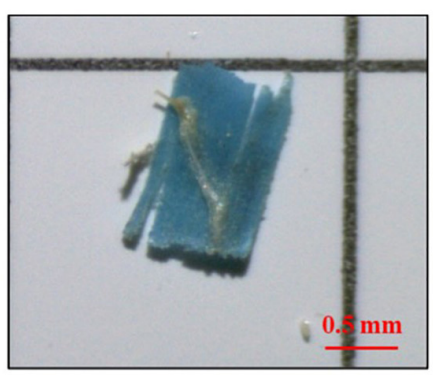

E

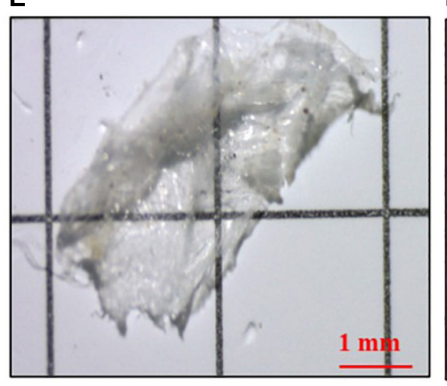

c

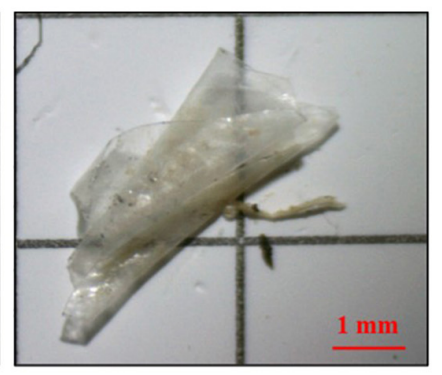

$\mathbf{F}$

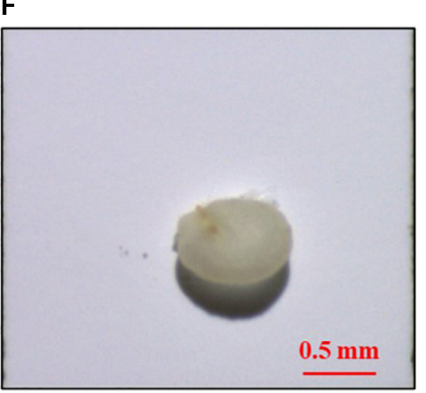

FIGURE 4 | Photographs of microplastics with different shapes under the stereomicroscope: fiber (A), fragment (B,C), film (D,E), and pellet (F).

layers. These results are consistent with those of previous studies on agricultural soils in Shanghai and Shandong, China (Liu et al., 2018; Yu et al., 2020). However, they are in contrast to those of Zhang et al. (2020) who found the abundance of microplastics at $20-30 \mathrm{~cm}\left(400\right.$ items $\left.\mathrm{kg}^{-1}\right)$ was much higher than that in the shallow soil layer $(0-20 \mathrm{~cm}, 100$ items $\mathrm{kg}^{-1}$ ) in suburban farm soils of Harbin, China. The differences in the vertical distributions of microplastics abundance in different studies may be related to many factors, including crop rotation, sampling depth, precipitation, and biological and mechanical disturbance.

Microplastics particles $<0.5 \mathrm{~mm}$ were predominant in this study (Figure 3), and the percentage of size distribution decreased with increased particle size. This may be ascribed to the gradual fragmentation of large pieces into small pieces by mechanical abrasion, high temperatures, and ultraviolet radiation, among other factors (Song et al., 2017; Piehl et al., 2018). The percentage of microplastics $<0.5 \mathrm{~mm}$ was significantly higher in non-mulched soils. This is because although the number of microplastics $<0.5 \mathrm{~mm}$ in mulched soils is greater than that in non-mulched soils, the total abundance is significantly lower in non-mulched soils, leading to a high percentage of microplastics $<0.5 \mathrm{~mm}$. The average size of microplastics in topsoil of the mulched and nonmulched farmland was significantly larger than that at deep soil. In addition, the percentage of small-sized microplastics (i.e., $<0.5 \mathrm{~mm}$ ) increased with increased soil depth, whereas that of large-sized particles (i.e., $2.0-5.0 \mathrm{~mm}$ ) decreased with increased soil depth (Figures 3A,C). This demonstrated that the mobility of smaller microplastics was higher than that of larger microplastics. These results are similar to those of $\mathrm{Yu}$ et al. (2020). This is partially because small-sized microplastics are liable to migrate to deeper soil layers under the influence of soil organisms; for example, earthworms can ingest smallsized microplastics and then excrete them with feces in deep soil channels (Huerta Lwanga et al., 2017). Small-sized microplastics are also more susceptible to soil erosion and runoff and can easily penetrate downward into deep soil layers through tiny cracks and pores (Bläsing and Amelung, 2018; Zhang et al., 2018). However, further research is needed on the transformation and migration of microplastics in soils.

\section{Morphology and Composition Characteristics of Microplastics in Maize Farmland Soils}

In this study, white (41.8\%) and transparent (38.0\%) were the dominant colors of microplastics in maize farmland soils, followed by yellow (17.5\%), blue (3.8\%), and black (1.9\%). This is consistent with previous studies (Piehl et al., 2018; Amrutha and Warrier, 2020; Yu et al., 2020), although Liu et al. (2018) found translucent and black microplastics dominated in agricultural soils. The diversity of color in microplastics is also a reflection of the diversity of pollution sources (Zhang D. D. et al., 2021). White and transparent microplastics are from a wide range of sources, including plastic packaging materials, plastic bags, and plastic film (Supplementary Figure 2). In addition, polychromatic microplastics may be weathered and discolored under the actions of water, heat, and light to form white microplastics (Galafassi et al., 2019; Liu et al., 2020). However, multicolor microplastics may be sourced from colorful plastic consumer products, plastic packaging, clothing, and cosmetics used in daily life.

Across all sample sites, the dominant shapes of microplastics were fragments (39.8\%), films (29.1\%), and 

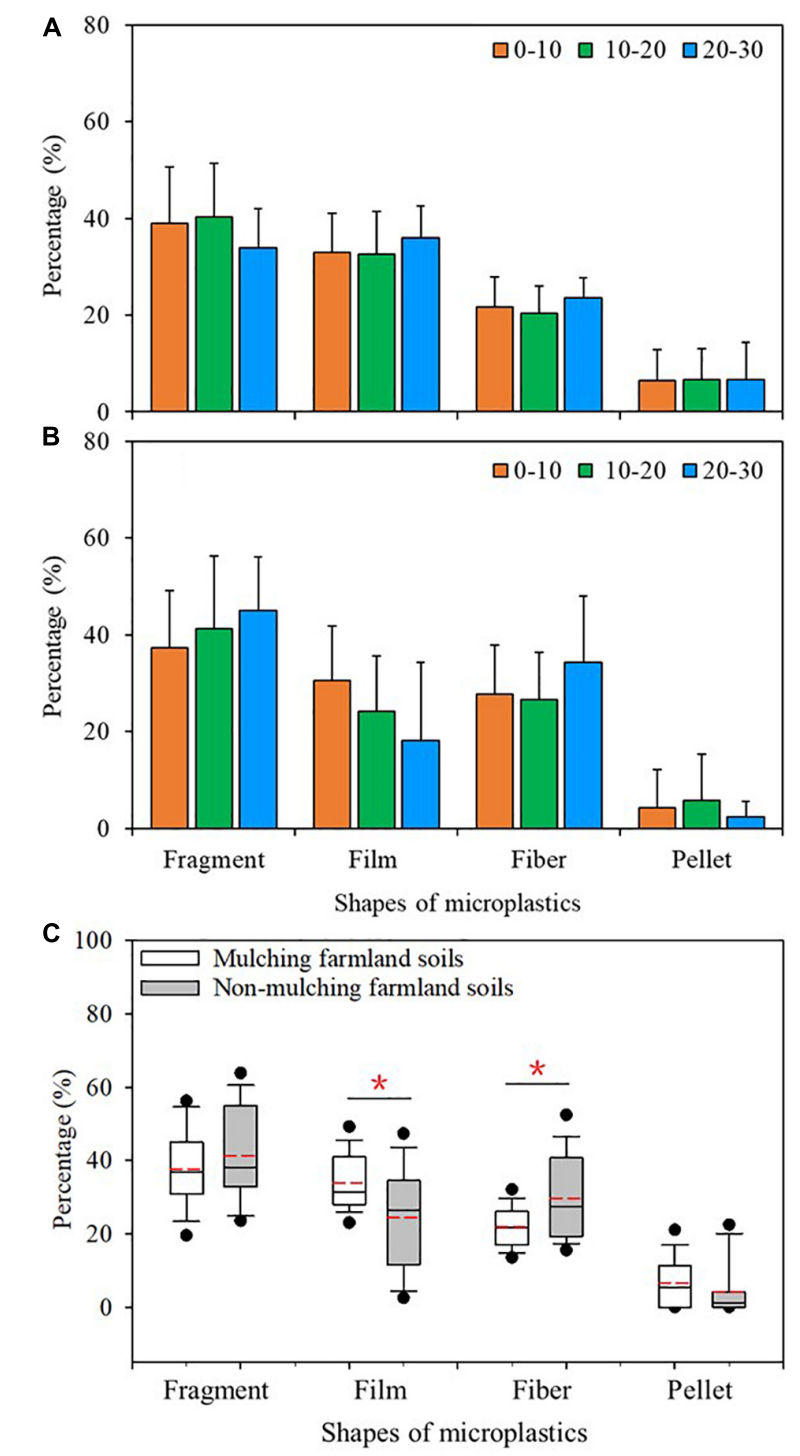

FIGURE 5 | Shape distribution of microplastics at different soil depths (cm) in maize farmlands. Percentages of different shapes of microplastics at different depths in (A) mulched and (B) non-mulched soils [Columns represent the mean ( $n=10$ in mulched soils and $n=5$ in non-mulched soils) and standard deviations]. (C) Percentages of different microplastics shapes in mulched $(n=30)$ and non-mulched $(n=15)$ soils $\left({ }^{*} p<0.05\right)$. Box-whisker diagrams show the average, 25th, 50th, and 75th percentiles.

fibers (25.7\%) (Figures 5A,B). The result was similar to some findings previously reported (Amrutha and Warrier, 2020; Ding et al., 2020; Zhou et al., 2020), whereas it was differed from the results in which microbeads or flakes microplastics are dominant types (Zhou et al., 2018; Chen et al., 2020). Such differences in dominant shapes are likely because the sources of microplastics are different in different study areas. Polymer types in fragments were mainly PP and PE, accounting for 66.4 and 32.7\%, respectively. Polypropylene is widely used in snack wrappers and food packaging, whereas PE is often used in plastic containers, food
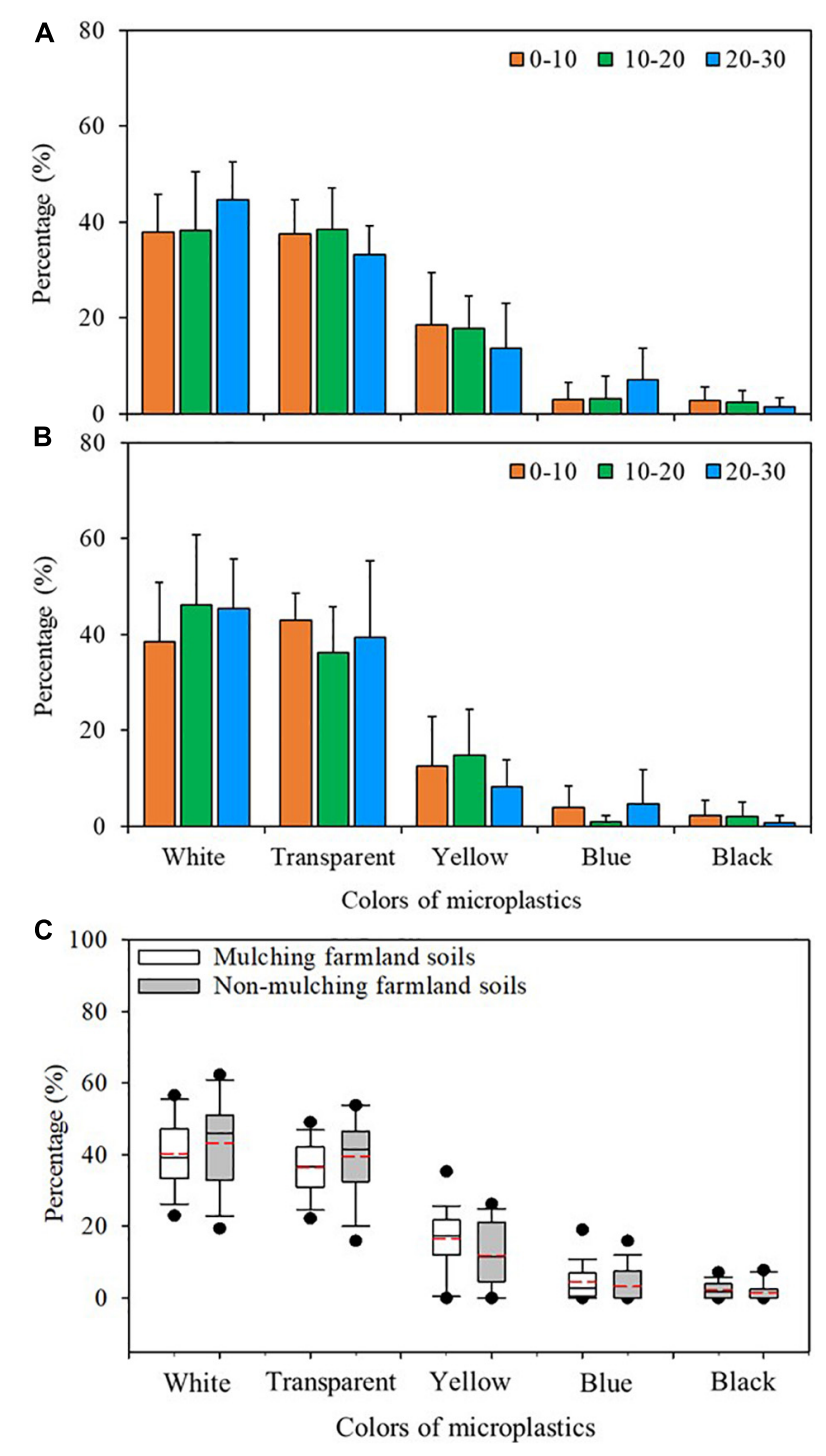

FIGURE 6 | Color distribution of microplastics at different soil depths (cm) in maize farmlands. Percentages of different colors of microplastics at different depths in (A) mulched and (B) non-mulched soils [Columns represent the mean ( $n=10$ in mulched soils and $n=5$ in non-mulched soils]. (C) Percentages of different microplastics colors in mulched $(n=30)$ and non-mulched ( $n=15$ ) soils. Box-whisker diagrams show the average, 25th, 50th, and 75th percentiles. 

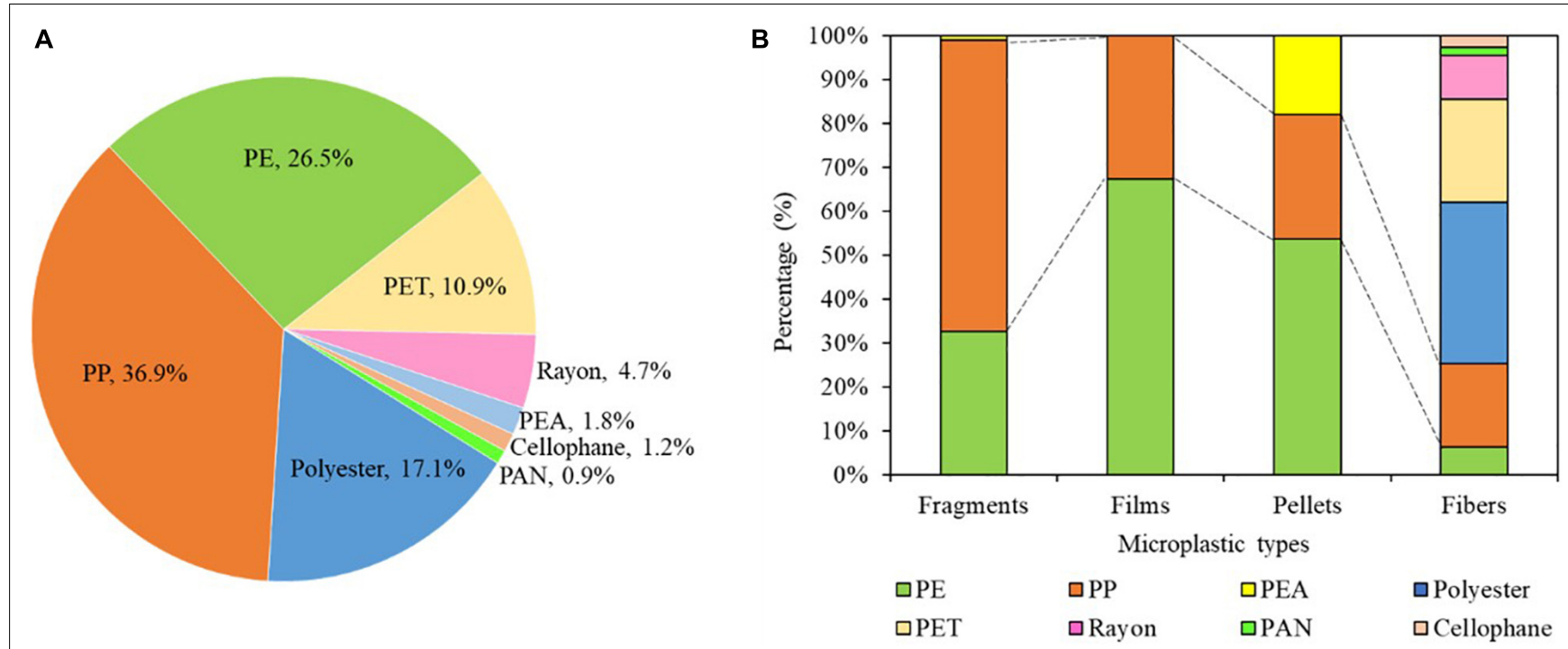

FIGURE 7 | Polymer composition of microplastics in maize farmland soils. (A) Overall percentages of different polymer compositions of microplastics. (B) Percentage contributions of different polymers of different shapes of microplastics. PP, polypropylene; PE, polyethylene; PET, poly(ethylene terephthalate); PEA, poly(ethyl acrylate); PAN, polyacrylonitrile/acrylic acid. Polymer composition was determined in 339 microplastics samples.

The polymer compositions of films included PE (67.4\%) and PP (32.6\%). The percentage of films was significantly higher in mulched soils (Figure 5C). In the present study, $\mu$-FTIR determinations indicated that PE served as the main polymer composition of agricultural plastic mulching films (commercial products) used in maize farmland soils with plastic film mulching. The thickness of plastics films used in agricultural production in China is generally $<0.006 \mathrm{~mm}$, much thinner than that used in Japan or Europe (i.e., $0.02 \mathrm{~mm}$ ), and therefore, films are easily torn during cultivation, and recycling is also difficult (Zhao et al., 2017). Although local farmers claimed they would recycle most plastic films after maize harvest, plastic films remaining in the farmlands may lose integrity and decompose into increasingly smaller plastic pieces of different sizes, of which some eventually form microplastics (Briassoulis et al., 2015; Steinmetz et al., 2016). Therefore, the application of plastic mulching film was a major contributor to films microplastics with a dominant component of PE in maize fields.

The polymer composition of fibers was widely variable in this study. They were composed primarily of polyester and PET; in addition, PP, rayon, PE, cellophane, and PAN were also identified in fibers. Notably, although polyester and PET materials are not widely adopted in agricultural production, they were found in maize farmland soils. These two polymers are mainly adopted in textile industries, and they have been detected at high contents in irrigation water that contains high levels of plastic fibers produced by laundry (Lusher et al., 2013; Zhou et al., 2020). Therefore, agricultural irrigation water may be an important pathway of fibers into the maize fields. In addition, atmospheric dry and wet deposition are also potential sources of fiber microplastics in soils (Dris et al., 2016; Leads and Weinstein, 2019; Liu et al., 2019). In this study, fibers showed substantially higher percentage in non-mulched soils.
This may be due to the small difference in fibers from the above sources between mulched and non-mulched soils, and the much lower abundance of microplastics in non-mulched soils, resulting in a relatively high proportion of fibers. Although the density of polyester and PET is slightly higher than that of saturated $\mathrm{NaCl}$, this study found that some PET and polyester fibers were extracted, similar findings were also reported by Li et al. (2021) and $\mathrm{Yu}$ et al. (2020). It may be attributed to that the density of microplastics was changed compared to theoretical plastics due to the changes of their physical and chemical properties and occurrence state in the actual environment (Scheurer and Bigalke, 2018). In addition, the combined action of the surface tension and buoyancy of small particles may make the saturated $\mathrm{NaCl}$ solution separate microplastics with slightly higher density (Li et al., 2021).

Pellets were a small percentage of the microplastics (5.4\%) in the study areas, and the main polymer compositions included PE, and PP, which was consistent with the results reported by Han et al. (2020). Microplastics are added as abrasives to many products, including toothpastes and hand and facial cleaners, which can be discharged into the environment through domestic sewage (Duis and Coors, 2016). According to studies in Norway, Switzerland, and the European Union, approximately $6 \%$ of liquid skin-cleaning products contain microplastics, of which $93 \%$ are composed of PE (Gouin et al., 2015). Therefore, the microbeads in personal cleaning and care products might be the source of pellet microplastics (Lee et al., 2013; Ding et al., 2020). In brief, the results of this study demonstrated the different sources of microplastics in farmland soils. Apart from plastic film, other agricultural plastic materials should also be paid more attention to avoid excessive accumulation of microplastics in soils. 


\section{CONCLUSION}

Microplastics pollution characteristics was revealed in typical maize farmland soils with and without long-term plastic film mulching. Mulched soils contained much higher abundances of microplastics than non-mulched soils. Microplastics were the most abundant and the largest in topsoil. Small microplastics $(<0.5 \mathrm{~mm})$ were dominant, and their percentage increased with increased soil depth, indicating that small-sized microplastics tended to migrate to deeper soil layers. Mulched soils had much lower proportion of microplastics $<0.5 \mathrm{~mm}$ than non-mulched soils. Microplastics were mainly fragments, films, and fibers, and mulched soils had much higher percentage of films and lower percentage of fibers than non-mulched soils. Polypropylene, $\mathrm{PE}$, and polyester were the dominated polymer compositions. In addition, the length of time with film mulching applied was greatly affect the accumulation of microplastics in soils. Overall, this study provides an important reference for future research on ecological risks of microplastics in agroecosystems. In future, more attention should be paid to related remediation and management strategies to reduce mulching-based microplastics pollution in agricultural soils, such as the development of biodegradable films and efficient recycle technologies.

\section{DATA AVAILABILITY STATEMENT}

The datasets presented in this study can be found in online repositories. The names of the repository/repositories and accession number(s) can be found in the article/ Supplementary Material.

\section{REFERENCES}

Ahmed, T., Shahid, M., Azeem, F., Rasul, I., Shah, A. A., Noman, M., et al. (2018). Biodegradation of plastics: current scenario and future prospects for environmental safety. Environ. Sci. Pollut. Res. 25, 7287-7298. doi: 10.1007/ s11356-018-1234-9

Amrutha, K., and Warrier, A. K. (2020). The first report on the source-to-sink characterization of microplastic pollution from a riverine environment in tropical India. Sci. Total Environ. 739:140377. doi: 10.1016/j.scitotenv.2020. 140377

Astner, A. F., Hayes, D. G., O’ Neill, H. O., Evans, B. R., Pingali, S. V., Urban, V. S., et al. (2019). Mechanical formation of micro- and nano-plastic materials for environmental studies in agricultural ecosystems. Sci. Total Environ. 685, 1097-1106. doi: 10.1016/j.scitotenv.2019.06.241

Avio, C. G., Gorbi, S., Milan, M., Benedetti, M., Fattorini, D., D’Errico, G., et al. (2015). Pollutants bioavailability and toxicological risk from microplastics to marine mussels. Environ. Pollut. 198, 211-222. doi: 10.1016/j.envpol.2014. 12.021

Barboza, L. G. A., Vethaak, A. D., Lavorante, B. R. B. O., Lundebye, A. K., and Guilhermino, L. (2018). Marine microplastic debris: an emerging issue for food security, food safety and human health. Mar. Pollut. Bull. 133, 336-348. doi: 10.1016/j.marpolbul.2018.05.047

Bläsing, M., and Amelung, W. (2018). Plastics in soil: analytical methods and possible sources. Sci. Total Environ. 612, 422-435. doi: 10.1016/j.scitotenv.2017. 08.086

Briassoulis, D., Babou, E., Hiskakis, M., and Kyrikou, I. (2015). Analysis of longterm degradation behaviour of polyethylene mulching films with pro-oxidants under real cultivation and soil burial conditions. Environ. Sci. Pollut. Res. 22, 2584-2598. doi: 10.1007/s11356-014-3464-9

\section{AUTHOR CONTRIBUTIONS}

JZ conducted the laboratory analysis, formal analysis, and wrote the manuscript. GZ and YC co-supervised the project and assisted with various methodological of the associated manuscript. XW, BL, and YM conducted the investigation. LX assisted with methodological. WD, XZ, and LS contributed to editing. All authors contributed to the article and approved the submitted version.

\section{FUNDING}

Financial supports from the China Agriculture Research System of the Ministry of Finance of the People's Republic of China and the Ministry of Agriculture and Rural Affairs of the People's Republic of China, Beijing Academy of Agriculture and Forestry Sciences (KJCX20210430 and YZS202001), Project funded by China Postdoctoral Science Foundation (2021M700493), Beijing Postdoctoral Research Foundation (2021-ZZ-135), and Research \& Demonstration Project of Water Conservation and Agricultural Non-point Source Pollution Control Technology in Chaohe River Basin (202008F016).

\section{SUPPLEMENTARY MATERIAL}

The Supplementary Material for this article can be found online at: https://www.frontiersin.org/articles/10.3389/fmars. 2021.800087/full\#supplementary-material

Chen, Y. L., Leng, Y. F., Liu, X. N., and Wang, J. (2020). Microplastic pollution in vegetable farmlands of suburb Wuhan, central China. Environ. Pollut. 257:113449. doi: 10.1016/j.envpol.2019.113449

Ding, L., Zhang, S. Y., Wang, X. Y., Yang, X. M., Zhang, C. T., Qi, Y. B., et al. (2020). The occurrence and distribution characteristics of microplastics in the agricultural soils of Shaanxi Province, in north-western China. Sci. Total Environ. 720:137525. doi: 10.1016/j.scitotenv.2020.137525

Dris, R., Gasperi, J., Saad, M., Mirande, C., and Tassin, B. (2016). Synthetic fibers in atmospheric fallout: a source of microplastics in the environment? Mar. Pollut. Bull. 104, 290-293. doi: 10.1016/j.marpolbul.2016.01.006

Duis, K., and Coors, A. (2016). Microplastics in the aquatic and terrestrial environment: sources (with a specific focus on personal care products), fate and effects. Environ. Sci. Eur. 28:2. doi: 10.1186/s12302-015-0069-y

Farrell, P., and Nelson, K. (2013). Trophic level transfer of microplastic: mytilus edulis (L.) to Carcinus maenas (L.). Environ. Pollut. 177, 1-3. doi: 10.1016/j. envpol.2013.01.046

Fuller, S., and Gautam, A. (2016). A procedure for measuring microplastics using pressurized fluid extraction. Environ. Sci. Technol. 50, 5774-5780. doi: 10.1021/ acs.est.6b00816

Galafassi, S., Nizzetto, L., and Volta, P. (2019). Plastic sources: a survey across scientific and grey literature for their inventory and relative contribution to microplastics pollution in natural environments, with an emphasis on surface water. Sci. Total Environ. 693:133499. doi: 10.1016/j.scitotenv.2019.07.305

Gong, J., and Xie, P. (2020). Research progress in sources, analytical methods, eco-environmental effects, and control measures of microplastics. Chemosphere 254:126790. doi: 10.1016/j.chemosphere.2020.126790

Gouin, T., Avalos, J., Brunning, I., Brzuska, K., and Wolf, T. (2015). Use of microplastic beads in cosmetic products in Europe and their estimated emissions to the North Sea environment. Sofw J. 141, 40-46. 
Han, L. H., Li, Q. L., Xu, L., Lu, A. X., Li, B. R., Gong, W. W., et al. (2020). Abundance and Distribution of Microplastics of Soils in Daliao River Basin. Asian J. Ecotoxicol. 15, 174-185. doi: 10.7524/AJE.1673-5897.20190911003

He, D. F., Luo, Y. M., Lu, S. B., Liu, M. T., Song, Y., and Lei, L. L. (2018). Microplastics in soils: analytical methods, pollution characteristics and ecological risks. Trends Anal. Chem. 109, 163-172. doi: 10.1016/j.trac.2018. 10.006

Hebei Statistics Bureau (2020). Hebei Statistical Yearbook 2020. Beijing: China Statistics Press.

Hidalgo-Ruz, V., Gutow, L., Thompson, R. C., and Thiel, M. (2012). Microplastics in the marine environment: a review of the methods used for identification and quantification. Environ. Sci. Technol. 46, 3060-3075. doi: 10.1021/es2031505

Horton, A. A., Walton, A., Spurgeon, D. J., Lahive, E., and Svendsen, C. (2017). Microplastics in freshwater and terrestrial environments: evaluating the current understanding to identify the knowledge gaps and future research priorities. Sci. Total Environ. 586, 127-141. doi: 10.1016/j.scitotenv.2017.01.190

Huang, Y., Liu, Q., Jia, W. Q., Yan, C. R., and Wang, J. (2020). Agricultural plastic mulching as a source of microplastics in the terrestrial environment. Environ. Pollut. 260:114096. doi: 10.1016/j.envpol.2020.114096

Huerta Lwanga, E., Gertsen, H., Gooren, H., Peters, P., Salánki, T., Ploeg, M. V. D., et al. (2017). Incorporation of microplastics from litter into burrows of Lumbricus terrestris. Environ. Pollut. 220, 523-531. doi: 10.1016/j.envpol.2016. 09.096

Huerta Lwanga, E., Gertsen, H., Gooren, H., Peters, P., Salánki, T., van der Ploeg, M., et al. (2016). Microplastics in the terrestrial ecosystem: implications for Lumbricus terrestris (Oligochaeta, Lumbricidae). Environ. Sci. Technol. 50, 2685-2691. doi: 10.1021/acs.est.5b05478

Hüffer, T., Weniger, A.-K., and Hofmann, T. (2018). Sorption of organic compounds by aged polystyrene microplastic particles. Environ. Pollut. 236, 218-225.

Leads, R. R., and Weinstein, J. E. (2019). Occurrence of tire wear particles and other microplastics within the tributaries of the Charleston Harbor Estuary. South Carolina, USA. Mar. Pollut. Bull. 145, 569-582. doi: 10.1016/j.marpolbul.2019. 06.061

Lee, J., Hong, S., Song, Y. K., Sang, H. H., Yong, C. J., Mi, J., et al. (2013). Relationships among the abundances of plastic debris in different size classes on beaches in South Korea. Mar. Pollut. Bull. 77, 349-354. doi: 10.1016/j. marpolbul.2013.08.013

Li, J., Zhang, K. N., and Zhang, H. (2018). Adsorption of antibiotics on microplastics. Environ. Pollut. 237, 460-467. doi: 10.1016/j.envpol.2018.02.050

Li, L. Z., Luo, Y. M., Li, R. J., Zhou, Q., Peijnenburg, W. J. G. M., Yin, N., et al. (2020). Effective uptake of submicrometer plastics by crop plants via a crack-entry mode. Nat. Sustain. 3, 929-937. doi: 10.1038/s41893-020-0567-9

Li, Q. L., Zeng, A. R., Jiang, X., and Gu, X. Y. (2021). Are microplastics correlated to phthalates in facility agriculture soil? J. Hazard. Mater. 412:125164. doi: 10.1016/j.jhazmat.2021.125164

Liu, M. T., Lu, S. B., Song, Y., Lei, L. L., Hu, J. N., Lv, W. W., et al. (2018). Microplastic and mesoplastic pollution in farmland soils in suburbs of Shanghai. China. Environ. Pollut. 242, 855-862. doi: 10.1016/j.envpol.2018. 07.051

Liu, P., Zhan, X., Wu, X. W., Li, J. L., Wang, H. Y., and Gao, S. X. (2020). Effect of weathering on environmental behavior of microplastics: properties, sorption and potential risks. Chemosphere 242:125193. doi: 10.1016/j.chemosphere.2019. 125193

Liu, X. M., Shi, H. H., Xie, B., Dionysiou, D. D., and Zhao, Y. P. (2019). Microplastics as both a sink and a source of bisphenol A in the marine environment. Environ. Sci. Technol. 53, 10188-10196. doi: 10.1021/acs.est. $9 \mathrm{~b} 02834$

Lusher, A. L., McHugh, M., and Thompson, R. C. (2013). Occurrence of microplastics in the gastrointestinal tract of pelagic and demersal fish from the English Channel. Mar. Pollut. Bull. 67, 94-99. doi: 10.1016/j.marpolbul.2012. 11.028

Lv, W. W., Zhou, W. Z., Lu, S. B., Huang, W. W., Yuan, Q., Tian, M. L., et al. (2019). Microplastic pollution in rice-fish co-culture system: a report of three farmland stations in Shanghai. China. Sci. Total Environ. 652, 1209-1218. doi: 10.1016/j.scitotenv.2018.10.321
Peng, G., Zhu, B., Yang, D., Su, L., Shi, H., and Li, D. (2017). Microplastics in sediments of the Changjiang Estuary, China. Environ. Pollut. 225, 283-290. doi: 10.1016/j.envpol.2016.12.064

Peng, G. Y., Xu, P., Zhu, B. S., Bai, M. Y., and Li, D. J. (2018). Microplastics in freshwater river sediments in Shanghai, China: a case study of risk assessment in mega-cities. Environ. Pollut. 234, 448-456. doi: 10.1016/j.envpol.2017. 11.034

Piehl, S., Leibner, A., Loder, M. G. J., Dris, R., Bogner, C., and Laforsch, C. (2018). Identification and quantification of macro- and microplastics on an agricultural farmland. Sci. Rep. 8:17950. doi: 10.1038/s41598-018-36172-y

Rillig, M. C. (2012). Microplastic in terrestrial ecosystems and the soil? Environ. Sci. Technol. 46, 6453-6454. doi: 10.1021/es302011r

Rillig, M. C., Ingraffia, R., De, S. M., and Anderson, A. (2017). Microplastic incorporation into soil in agroecosystems. Front. Plant Sci. 8:1805. doi: 10.3389/ fpls.2017.01805

Rillig, M. C., and Lehmann, A. (2020). Microplastics in terrestrial ecosystems. Science 368, 1430-1431. doi: 10.1126/science.abb5979

Scheurer, M., and Bigalke, M. (2018). Microplastics in Swiss floodplain soils. Environ. Sci. Technol. 52, 3591-3598. doi: 10.1021/acs.est.7b06003

Sighicelli, M., Pietrelli, L., Lecce, F., Iannilli, V., Falconieri, M., Coscia, L., et al. (2018). Microplastic pollution in the surface waters of Italian Subalpine Lakes. Environ Pollut. 236, 645-651.

Song, Y. K., Hong, S. H., Jang, M., Han, G. M., Jung, S. W., and Shim, W. J. (2017). Combined effects of UV exposure duration and mechanical abrasion on microplastic fragmentation by polymer type. Environ. Sci. Technol. 51, 4368-4376. doi: 10.1021/acs.est.6b06155

Statista (2020). Available online at: https://www.statista.com/statistics/282732/ global-production- of plasticssince-1950/, 2020 (accessed April 2021).

Steinmetz, Z., Wollmann, C., Schaefer, M., Buchmann, C., David, J., Troeger, J., et al. (2016). Plastic mulching in agriculture. Trading short-term agronomic benefits for long-term soil degradation? Sci. Total Environ. 550, 690-705. doi: 10.1016/j.scitotenv.2016.01.153

Sul, J. A. I. D., and Costa, M. F. (2014). The present and future of microplastic pollution in the marine environment. Environ. Pollut. 185, 352-364. doi: 10. 1016/j.envpol.2013.10.036

Tang, G., Liu, M., Zhou, Q., He, H., Chen, K., and Zhang, H. (2018). Microplastics and polycyclic aromatic hydrocarbons (PAHs) in Xiamen coastal areas: implications for anthropogenic impacts. Sci. Total Environ. 634, 811-820. doi: 10.1016/j.scitotenv.2018.03.336

Turner, A., and Holmes, L. A. (2015). Adsorption of trace metals by microplastic pellets in fresh water. Environ. Chem. 12, 600-610. doi: 10.1071/EN14143

Vaughan, R., Turner, S. D., and Rose, N. L. (2017). Microplastics in the sediments of a UK urban lake. Environ. Pollut. 229, 10-18. doi: 10.1016/j.envpol.2017.05.057

Wang, J., Li, J. Y., Liu, S. T., Li, H. Y., Chen, X. C., Peng, C., et al. (2020). Distinct microplastic distributions in soils of different land-use types: a case study of Chinese farmlands. Environ. Pollut. 269:116199. doi: 10.1016/j.envpol. 2020.116199

Wang, W., and Wang, J. (2018). Different partition of polycyclic aromatic hydrocarbon on environmental particulates in freshwater: microplastics in comparison to natural sediment. Ecotoxicol. Environ. Saf. 147, 648-655. doi: 10.1016/j.ecoenv.2017.09.029

Yang, J., Li, R. J., Zhou, Q., Li, L. Z., Tu, C., Zhao, X. Y., et al. (2020). Abundance and morphology of microplastics in an agricultural soil following long-term repeated application of pig manure. Environ Pollut. 272:116028. doi: 10.1016/ j.envpol.2020.116028

Yu, L., Zhang, J. D., Liu, Y., Chen, L. Y., Tao, S., and Liu, W. X. (2020). Distribution characteristics of microplastics in agricultural soils from the largest vegetable production base in China. Sci. Total Environ. 756:143860. doi: 10.1016/j. scitotenv.2020.143860

Zhang, D. D., Fraser, M. A., Huang, W., Ge, C. J., Wang, Y., Zhang, C. F., et al. (2021). Microplastic pollution in water, sediment, and specific tissues of crayfish (Procambarus clarkii) within two different breeding modes in Jianli, Hubei province, China. Environ Pollut. 272:115939. doi: 10.1016/j.envpol.2020.115939

Zhang, J. J., Chen, Y. H., Wang, X. X., Ni, X. H., Liu, D. S., Li, L. X., et al. (2021). A review of microplastics in the soil environment. Chin. J. Eco Agric. 29, 937-952. doi: 10.13930/j.cnki.cjea.200915 
Zhang, G. S., and Liu, Y. F. (2018). The distribution of microplastics in soil aggregate fractions in southwestern China. Sci. Total Environ. 642, 12-20. doi: 10.1016/j.scitotenv.2018.06.004

Zhang, J. J., He, P., Xu, X. P., Wang, Y. L., Jia, L. L., Cui, R. Z., et al. (2017). Nutrient expert improves nitrogen efficiency and environmental benefits for summer maize in China. Agron. J. 109, 1082-1090. doi: 10.2134/agronj2016.08.0477

Zhang, S., Yang, X., Gertsen, H., Peters, P., Salánki, T., and Geissen, V. (2018). A simple method for the extraction and identification of light density microplastics from soil. Sci. Total Environ. 616, 1056-1065. doi: 10.1016/j. scitotenv.2017.10.213

Zhang, S. L., Liu, X., Hao, X. H., Wang, J. Q., and Zhang, Y. (2020). Distribution of low-density microplastics in the mollisol farmlands of northeast China. Sci. Total Environ. 708:135091. doi: 10.1016/j.scitotenv.2019.135091

Zhao, Y., Chen, X., Wen, H., Zheng, X., Niu, Q., and Kang, J. (2017). Research status and prospect of control technology for residual plastic film pollution in farmland. Trans. Chin. Soc. Agric. 48, 1-14. doi: 10.6041/j.issn.1000-1298.2017. 06.001

Zhou, B. Y., Wang, J. Q., Zhang, H. B., Shi, H. H., Fei, Y. F., Huang, S. Y., et al. (2020). Microplastics in agricultural soils on the coastal plain of Hangzhou Bay, East China: multiple sources other than plastic mulching film. J. Hazard. Mater. 388:121814. doi: 10.1016/j.jhazmat.2019.121814

Zhou, Q., Zhang, H. B., Fu, C. C., Zhou, Y., Dai, Z. F., Li, Y., et al. (2018). The distribution and morphology of microplastics in coastal soils adjacent to the Bohai Sea and the Yellow Sea. Geoderma 322, 201-208. doi: 10.1016/j.geoderma. 2018.02.015
Zhou, Q., Zhang, H. B., Zhou, Y., Li, Y., Xue, Y., Fu, C. C., et al. (2016). Separation of microplastics from a coastal soil and their surface microscopic features. Sci. Bull. 61, 1604-1611. doi: 10.1360/N97201501098

Zubris, K. A., and Richards, B. K. (2005). Synthetic fibers as an indicator of land application of sludge. Environ. Pollut. 138, 201-211. doi: 10.1016/j.envpol.2005. 04.013

Conflict of Interest: The authors declare that the research was conducted in the absence of any commercial or financial relationships that could be construed as a potential conflict of interest.

Publisher's Note: All claims expressed in this article are solely those of the authors and do not necessarily represent those of their affiliated organizations, or those of the publisher, the editors and the reviewers. Any product that may be evaluated in this article, or claim that may be made by its manufacturer, is not guaranteed or endorsed by the publisher.

Copyright (C) 2021 Zhang, Zou, Wang, Ding, Xu, Liu, Mu, Zhu, Song and Chen. This is an open-access article distributed under the terms of the Creative Commons Attribution License (CC BY). The use, distribution or reproduction in other forums is permitted, provided the original author(s) and the copyright owner(s) are credited and that the original publication in this journal is cited, in accordance with accepted academic practice. No use, distribution or reproduction is permitted which does not comply with these terms. 